\title{
Enhancement of radiotherapy efficacy by miR-200c-loaded gelatinase-stimuli PEG-Pep-PCL nanoparticles in gastric cancer cells
}

This article was published in the following Dove Press journal:

International Journal of Nanomedicine

13 May 2014

Number of times this article has been viewed

\author{
Fang-bo Cui ${ }^{1, *}$ \\ Qin Liu',** \\ Ru-Tian $\mathrm{Li}^{\prime}$ \\ Jie Shen' \\ Pu-yuan Wu' \\ Li-Xia Yu' \\ Wen-jing $\mathrm{Hu}^{\prime}$ \\ Feng-lei $\mathrm{Wu}^{2}$ \\ Chun-Ping Jiang ' \\ Guo-feng Yue ${ }^{2}$ \\ Xiao-Ping Qian' \\ Xi-Qun Jiang ${ }^{3}$ \\ Bao-Rui Liu'
}

'The Comprehensive Cancer Center of Drum-Tower Hospital, Medical School of Nanjing University and

Clinical Cancer Institute of Nanjing

University, Nanjing, ${ }^{2}$ Nanjing Medical

University, ${ }^{3}$ Laboratory of Mesoscopic

Chemistry and Department of

Polymer Science and Engineering,

College of Chemistry and Chemical

Engineering, Nanjing University,

Nanjing, People's Republic of China

*These authors contributed equally to this work

\begin{abstract}
Radiotherapy is the main locoregional control modality for many types of unresectable tumors, including gastric cancer. However, many patients fail radiotherapy due to intrinsic radioresistance of cancer cells, which has been found to be strongly associated with cancer stem cell (CSC)-like properties. In this study, we developed a nanoparticle formulation to deliver miR$200 \mathrm{c}$, which is reported to inhibit CSC-like properties, and then evaluated its potential activity as a radiosensitizer. miR-200c nanoparticles significantly augmented radiosensitivity in three gastric cancer cell lines (sensitization enhancement ratio 1.13-1.25), but only slightly in GES-1 cells (1.06). In addition to radioenhancement, miR-200c nanoparticles reduced the expression of CD44, a putative CSC marker, and the percentage of CD44+ $\mathrm{BGC} 823$ cells. Meanwhile, other CSC-like properties, including invasiveness and resistance to apoptosis, could be suppressed by miR-200c nanoparticles. CSC-associated radioresistance mechanisms, involving reactive oxygen species levels and DNA repair capacity, were also attenuated. We have demonstrated that miR-200c nanoparticles are an effective radiosensitizer in gastric cancer cells and induce little radiosensitization in normal cells, which suggests that they are as a promising candidate for further preclinical and clinical evaluation.
\end{abstract}

Keywords: radiosensitizer, miR-200c, gelatinase-stimuli nanoparticles, cancer stem cell-like properties, gastric cancer

\section{Introduction}

Gastric cancer remains a severe public health problem throughout the world, especially in the People's Republic of China. ${ }^{1}$ Radiotherapy is the main locoregional control modality for unresectable gastric cancer, but the intrinsic radioresistance of gastric cancer cells leads to failure of radiotherapy in many patients. ${ }^{2}$ The response of tumors to radiotherapy has been found to be highly dependent on inactivation or survival of cancer stem cells (CSCs) in several types of cancer, ${ }^{3-5}$ including gastric cancer. ${ }^{6}$ Recent studies suggest that CSCs might be more intrinsically radioresistant than their counterparts without stem-like properties. ${ }^{4,7}$ Radioresistance of CSCs may also be due to more effective DNA repair capacity ${ }^{4,7,8}$ and reduced reactive oxygen species levels. ${ }^{3}$ It was confirmed that inhibiting CSC properties would have the potential to improve radiotherapy response. ${ }^{9,10} \mathrm{CSC}$-targeted agents, including some herbal extracts, ${ }^{11}$ salinomycin, ${ }^{12}$ and microRNAs (miRNAs), have shown promise as candidates for enhancement of the effects of radiotherapy.

miRNAs are a class of small noncoding regulatory RNAs that regulate the translation of specific mRNAs. Several studies have revealed an association between the miR-200 family and CSCs. miR-200c, a major member of this family, can inhibit 
CSCs by regulating critical CSC-like properties, such as self-renewal, invasiveness, and differentiation. ${ }^{13-15}$

In fact, members of the miR-200 family are well known to be crucial modulators of epithelial-mesenchymal transition (EMT) by suppressing expression of ZEB1 and ZEB2, two transcriptional repressors of E-cadherin. ${ }^{16}$ Several reports indicate that emergence of CSCs occurs in part as the result of EMT. ${ }^{17}$ The EMT process results in acquisition of mesenchymal characteristics, including motility, invasiveness, chemoresistance, and radioresistance. Intriguingly, these characteristics triggered by EMT overlap with CSC-like properties, confirming an association between EMT, CSCs, and therapeutic failure. Furthermore, there is evidence showing that EMT may directly induce radioresistance. ${ }^{18}$ Generation of CSCs and the EMT process may be two coalitional and overlapping factors affecting progression of cancer and therapeutic failure. ${ }^{19}$

Because of these functions, miR-200c has the potential to promote radiosensitivity in cancer cells by inhibiting CSClike properties and repressing EMT. However, successful application of miRNAs has been impeded by instability and inefficient uptake by cells. To solve these problems, nanoparticle delivery systems have been investigated and could improve the physical stability of miRNA structures, protect miRNA from nuclease degradation, and aid in effective cellular uptake. ${ }^{20}$ Moreover, nanoparticles have the ability to achieve tumor-targeted drug delivery via the enhanced permeability and retention effect and a targeting strategy. Nanoparticles could deliver functional miRNAs into living cancer cells efficiently and selectively.

In a previous study, we developed gelatinase-stimuli nanoparticles for tumor-targeted drug delivery. ${ }^{21-23}$ These nanoparticles consisted of gelatinase-cleavage peptide with a poly(ethylene glycol) (PEG) and poly ( $\varepsilon$-caprolactone) (PCL)based structure. This structure was confirmed to be transformed by gelatinases, which are secreted exogenously by cells, facilitating drug release and cellular uptake. We then developed miR-200c-loaded nanoparticles based on the gelatinase-stimuli strategy, and evaluated their main characteristics. In the present study, we investigated whether miR-200c-loaded gelatinasestimuli nanoparticles (miR-200c nanoparticles) can serve as radiosensitizers. miR-200c nanoparticles were engineered, and we then measured the radioenhancement efficacy of these nanoparticles in gelatinase-overexpressing gastric cancer cells and gelatinase-deficient gastric epithelial cells using clonogenic and MTT (3-[4,5-dimethylthiazol-2-yl]-2,5 diphenyltetrazolium bromide) assays. Expression of the CSC marker CD44 and of the epithelial marker E-cadherin was detected by real-time poly- merase chain reaction (PCR) and Western blot in the BGC823 human gastric adenocarcinoma cancer cell line. The percentage of $\mathrm{CD} 44^{+} \mathrm{BGC} 823$ cells was detected by flow cytometry. CSClike properties and CSC-associated radioresistance mechanisms were also investigated.

\section{Materials and methods Cell culture and ionizing radiation}

Three human gastric adenocarcinoma cell lines (BGC823, SGC7901, and MKN45) and an immortalized human gastric mucosa cell line (GES-1) were purchased from the Shanghai Institute of Cell Biology (Shanghai, People's Republic of China) and maintained in Roswell Park Memorial Institute 1640 medium with $10 \%$ calf bovine serum, 50 units $/ \mathrm{mL}$ penicillin, and 50 units $/ \mathrm{mL}$ streptomycin in $5 \% \mathrm{CO}_{2}$ at $37^{\circ} \mathrm{C}$. Radiotherapy was administered in vitro using a $4 \mathrm{MeV}$ electron beam linear accelerator (Elekta, Stockholm, Sweden).

\section{Preparation of miR-200c nanoparticles and cellular uptake assay}

PEG-Pep-PCL copolymers were synthesized via ring opening copolymerization and amidation. miR-200c-loaded nanoparticles were then prepared using a double-emulsion solvent evaporation technique as previously described by our group. ${ }^{23}$ For the cellular uptake study, Rhodamine-B, which has high water-solubility, was chosen as a fluorescence probe to track the location of the nanoparticles. RhodamineB-loaded gelatinase-stimuli nanoparticles were also obtained by a double-emulsion solvent evaporation technique. Next, $10^{5}$ BGC823 or GES-1 cells were seeded in 6-well plates and incubated with Rhodamine-B-loaded gelatinase-stimuli nanoparticles. The fluorescent signals were then imaged using a fluorescence microscope (Olympus, Tokyo, Japan).

\section{Cell viability assay}

Cell viability after treatment with blank, free miR-200c, or miR-200c nanoparticles was evaluated by MTT assay, performed as described elsewhere. ${ }^{23}$ The four abovementioned cell lines were treated with drugs for 24 hours, followed by exposure to $6 \mathrm{~Gy}$ ionizing radiation. Cell proliferation was determined by MTT assay 7 days post ionizing radiation.

\section{Clonogenic survival assay}

Cells were treated with subtoxic concentrations of miR-200c nanoparticles (cell proliferation inhibition $<15 \%, 128 \mu \mathrm{mol} / \mathrm{L}$ for BGC823 and GES-1, $512 \mu \mathrm{mol} / \mathrm{L}$ for SGC7901, and $256 \mu \mathrm{mol} / \mathrm{L}$ for MKN45, respectively, Figure S1) for 24 hours and then exposed to increasing doses of ionizing radiation 
$(0,2,4,6$, and 8 Gy). The clonogenic survival assay and determination of the sensitizer enhancement ratio (SER) were performed as described previously (Figure S2). ${ }^{24}$ BGC823 cells were mock-treated or pretreated with nanoparticles for 24 hours and then exposed to 6 Gy of ionizing radiation. Twenty-four hours following treatment, several assays were performed as described in the following sections.

\section{Transwell invasion assay}

Cell invasiveness was assessed using the Transwell migration assay. Six-well Transwell chambers (Corning Inc., New York, NY, USA) containing $8 \mu \mathrm{m}$ pores were prepared in advance by coating with $700 \mu \mathrm{L}$ Matrigel (BD Bioscience, San Diego, CA, USA) on ice. The Transwell migration assay was then performed as described elsewhere. ${ }^{25}$ Briefly, BGC823 cells that had been exposed to ionizing radiation with or without pretreatment with blank nanoparticles or miR-200c nanoparticles for 24 hours were collected and suspended in serumfree Roswell Park Memorial Institute 1640 medium (with a cell density of $2 \times 10^{5}$ cells $/ \mathrm{mL}$ ). A $1 \mathrm{~mL}$ cell suspension was added to the upper Transwell chamber and $1 \mathrm{~mL}$ of Roswell Park Memorial Institute 1640 medium with 15\% calf bovine serum was added to the lower chamber. After 24 hours of incubation, the cells remaining on the upper surface of the chamber were removed, and the migrated cells adhering to the lower surface were fixed with paraformaldehyde and stained with Giemsa stain. The numbers of migrated cells in at least five random microscopic fields per chamber were counted under a light microscope at a magnification of $200 \times$.

\section{Real-time PCR assays}

Real-time PCR assays to measure mRNA expression levels of miR-200c, E-cadherin, and CD44 were performed as published elsewhere. ${ }^{23}$ Total RNA was harvested from cells using TRIzol ${ }^{\circledR}$ reagent (Life Technologies, Carlsbad, CA, USA). The isolated RNA was reverse transcribed into complementary DNA using an ExScript ${ }^{\mathrm{TM}}$ RT reagent kit (Takara Bio Inc, Otsu, Japan) according to the manufacturer's instructions. The reaction was allowed to proceed at $25^{\circ} \mathrm{C}$ for 10 minutes and then at $37^{\circ} \mathrm{C}$ for one hour. For normalization, reverse transcription PCR was done on complementary DNA using eukaryotic 18S rRNA endogenous control primers and a FAM-MGB probe (Applied Biosystems, Foster City, CA, USA). A TaqMan miRNA reverse transcription kit was used to generate complementary DNA for PCR reaction in conjunction with a miR-200c-specific primer and probe (Applied Biosystems, assay ID 002300). The reverse transcription primer for miR-200c is a hairpin primer that is specific for mature miRNA and does not bind to the precursor molecules. For validation of the microarray data, SYBR Green real-time reverse transcription PCR was done using primers specific for E-cadherin and CD44. The specific primer sequences are shown in Figure S3. Relative mRNA and miRNA levels were calculated using the comparative $\mathrm{Ct}$ method $(\Delta \Delta \mathrm{Ct})$.

\section{Western blot assay}

Proteins in the cell culture supernatant were used directly for matrix metalloproteinase-2/9 detection, and cell extracts were prepared in suitable lysis buffer. Each sample was measured by Western blot assay as indicated. In brief, each sample prepared with loading buffer was separated by sodium dodecyl sulfate polyacrylamide gel electrophoresis at a constant voltage and electrotransferred onto polyvinylidene difluoride membranes (Millipore, Billerica, MA, USA). The membranes were then blocked for one hour at room temperature and incubated with a primary antibody overnight at $4^{\circ} \mathrm{C}$. The membranes were washed with TBS-T buffer three times and then incubated with a secondary antibody conjugated with horseradish peroxidase (Cell Signaling Technology, Beverly, MA, USA) for one hour. After washing three times, the blots were visualized using an enhanced chemiluminescence method (Millipore). Rabbit monoclonal antibodies against matrix metalloproteinase2/9, CD44, and E-cadherin were purchased from Abcam (Cambridge, UK) and rabbit monoclonal antibodies against caspase-3, caspase-8, caspase-9, and $\gamma$-H2AX from Cell Signaling Technology.

\section{Flow cytometry assays}

Reactive oxygen species generation, CD44 expression, and apoptosis were measured by flow cytometry. Intracellular reactive oxygen species levels were detected using the membrane-permeable fluorescent probe, $2^{\prime}, 7^{\prime}$-dichlorofluorescin diacetate (Beyotime Institute of Biotechnology, Shanghai, People's Republic of China). For the CD44 detection assay, cells were incubated with anti-CD44 phycoerythrin (eBioscience, San Diego, CA, USA). Immunoglobulin G-phycoerythrin was used as an isotype control. For the apoptosis assay, cells were costained with Alexa Fluor 488 Annexin V and propidium iodide (Life Technologies) after 72 hours of treatment, and then measured according to the manufacturer's instructions.

\section{Statistical analysis}

The data are shown as the mean \pm standard deviation. Comparisons between groups were evaluated by the 
Student's $t$-test. $P<0.05$ was accepted as being statistically significant.

\section{Results}

\section{Cellular uptake of gelatinase-stimuli nanoparticles was dependent on gelatinase expression}

miR-200c nanoparticles were prepared as described, and were found to have an average size of $170.4 \pm 0.80 \mathrm{~nm}$. Their surface charge (zeta potential) was $-2.4 \pm 0.02 \mathrm{mV}$ and their polydispersity index was about 0.094 . It has been reported that the miR-200c-loaded nanoparticles delivered miR-200c into BGC823 cells and showed sustained expression for 9 days. $^{23}$

In previous studies, we found that intracellular and excretory gelatinase (matrix metalloproteinase-2/9) levels are much higher in BGC823, SGC7901, and MKN45 cells than in GES-1 cells. Matrix metalloproteinase- $2 / 9$ could not be detected in the culture supernatant of GES-1 cells. ${ }^{26}$ Using a cellular uptake assay, we found that the fluorescence of Rhodamine-B-loaded gelatinase-stimuli nanoparticles in gelatinase-overexpressing BGC823 cells was significantly stronger than that in gelatinasedeficient GES-1 cells (Figure 1A).

After 24 hours of treatment with blank nanoparticles, free miR-200c, or miR-200c nanoparticles, we evaluated miR200c expression in BGC823 and GES-1 cells by real-time PCR (Figure 1B). Free miR-200c had a negligible effect on intracellular miR-200c expression in both BGC823 cells (1.3fold over control) and GES-1 cells (1.2-fold over control), in comparison with the other groups, significant miR-200c overexpression (40.3-fold over control) was observed in BGC823 cells treated with miR-200c nanoparticles. Meanwhile, only a 3.7-fold increase in miR-200c expression was observed in GES-1 cells treated with miR-200c nanoparticles. Overall, these results show that the nanoparticles delivered miR-200c into BGC823 cells efficiently, but not into GES-1 cells.

\section{miR-200c nanoparticles enhance} radiosensitivity in gastric cancer cells, but only slightly in gastric mucosa cells

Cell survival curves obtained from the clonogenic survival assay are shown in Figure 2A. Survival curves for the four cell lines treated with blank nanoparticles or free miR-200c almost overlapped those for cells treated with ionizing radiation alone. D0 values were calculated by a multitarget singlehit model. Sensitizer enhancement ratio (SER) was calculated as D0 ratio between combination treatment and IR alone. As shown in Figure 2B, treatment with blank nanoparticles or free miR-200c did not induce a significant increase in D0 $(P>0.05)$ in any of the cell lines when compared with cells treated with ionizing radiation alone.

However, compared with the groups that received ionizing radiation alone, D0 of the miR-200c nanoparticles and ionizing radiation group increased significantly in all three gastric cancer cell lines (Figure 2B, $P<0.01$ in BGC823 and SGC7901 cells, $P<0.05$ in MKN45 cells). The SER value was 1.25 for BGC823, 1.24 for SGC7901, and 1.13 for MKN45. However, treatment with miR-200c nanoparticles did not cause a statistically significant increase in D0 in the GES-1 cell line $(P>0.05)$, for which the SER value was 1.06. These results indicate that miR-200c nanoparticles enhance radiosensitivity significantly in gastric cancer cells, but only slightly in normal gastric mucosa cells.

The MTT assay also confirmed the selective radioenhancement achieved by miR-200c nanoparticles. The miR-200c nanoparticles sensitized BGC823 cells to 6 Gy of ionizing radiation and an additional $20.9 \%(P=0.011)$ increased proliferation inhibition compared with cells exposed to ionizing radiation alone. Similar results were observed in SGC7901 (20.5\%, $P<0.01)$ and MKN45 cells $(18.6 \%, P<0.01)$, but the increase in GES-1 cells was only $5.4 \%(P=0.192$, Figure 2C).

\section{miR-200c nanoparticles affected CSC markers and EMT}

First, we determined the effects of the combination of miR200c nanoparticles and ionizing radiation on CD44 by flow cytometry. Flow cytometry showed a significant decrease in $\mathrm{CD} 44^{+}$cell numbers when cells were treated with a combination of miR-200c nanoparticles and ionizing radiation (Figure 3A). Reductions in CD44 mRNA and protein expression levels were also confirmed in the group treated with a combination of miR-200c nanoparticles and ionizing radiation (Figure 3B).

We found that E-cadherin mRNA and protein expression levels were upregulated by miR-200c nanoparticles (Figure 3B). miR-200c is known to be an EMT inhibitor by suppressing E-cadherin. These results indicate that the basic functions of miR-200c were maintained in the nanoparticle formulation.

\section{miR-200c nanoparticles suppressed CSCs-like properties}

As shown in Figure 4A, the numbers of cells invading through the membrane into the lower compartment of the chamber in the Transwell invasion assay were 112.6 $\pm 8.0,83.7 \pm 6.1$, $81.7 \pm 8.7,35.7 \pm 6.0$ in the control, ionizing radiation, ionizing 
A

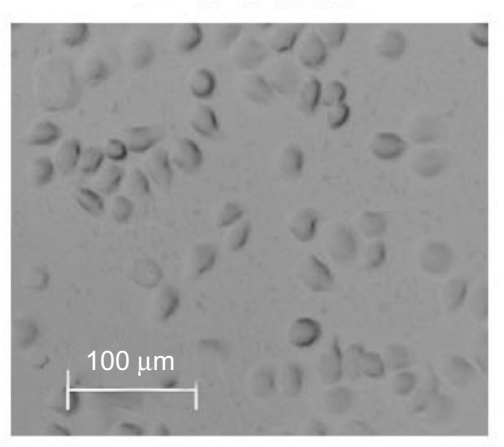

a

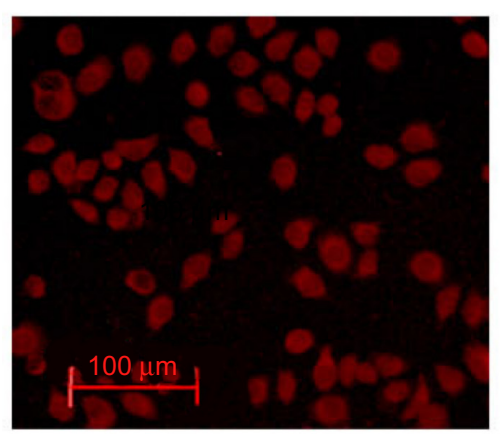

C

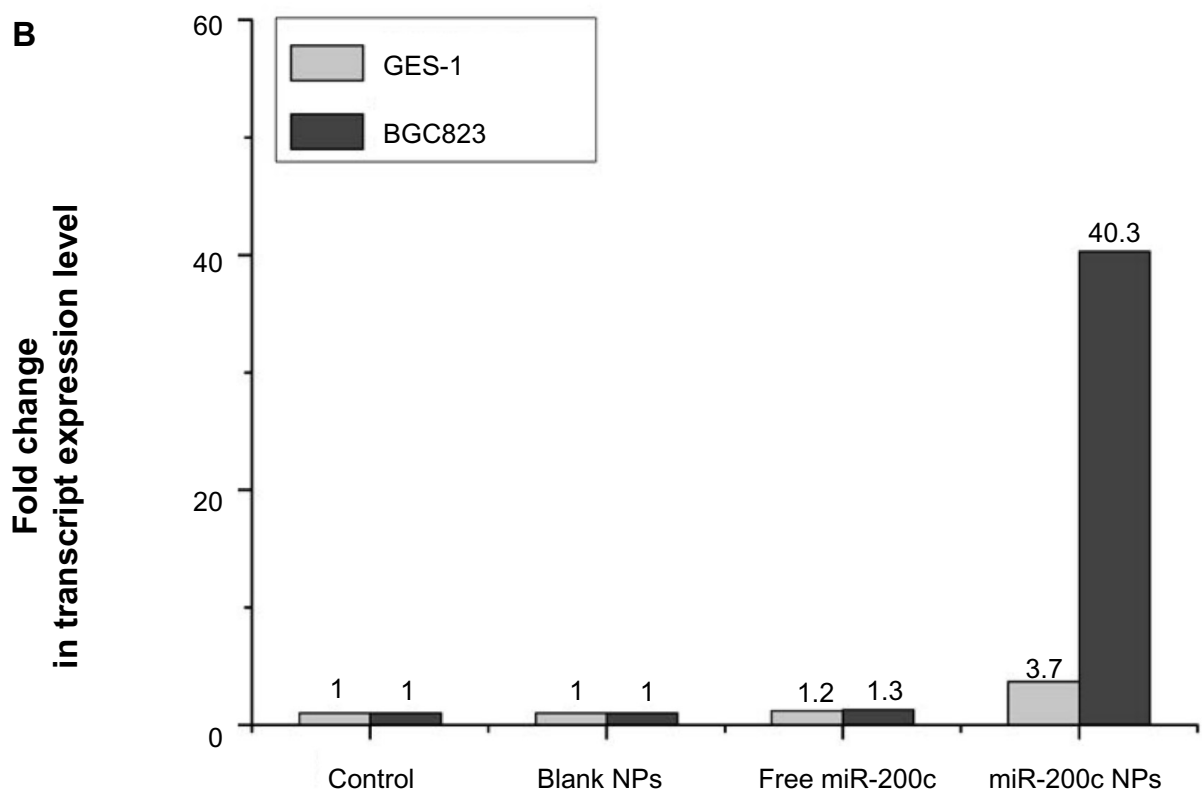

GES-1

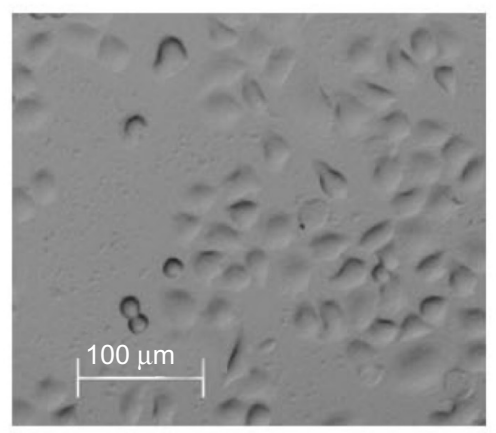

b

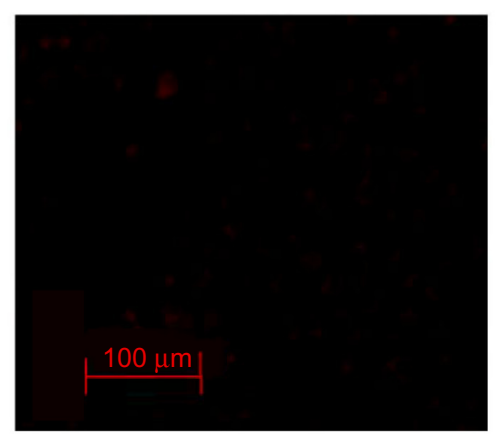

d

Figure I Cellular uptake of gelatinase-stimuli nanoparticles was dependent on expression of gelatinases.

Notes: (A) In a cellular uptake study, Rhodamine-B was used as a fluorescent marker to assess the efficiency of nanoparticle uptake by gelatinase-overexpressing cells BGC823 and gelatinase-deficient GES-I cells. BGC823 and GES-I cells ( $10^{5}$ per well) were seeded in 6-well plates and incubated with $500 \mu \mathrm{L}$ of medium containing coumarin6-loaded nanoparticles for 6 hours at $37^{\circ} \mathrm{C}$. The fluorescent signals from the cells were then imaged using a fluorescence microscope (Olympus, Tokyo, Japan). Microscopic images of BGC823 and GES-I cells incubated with Rhodamine-B loaded nanoparticles are shown in bright field (a and $\mathbf{b})$ and in fluorescent field (c and d). The fluorescence of Rhodamine-B-loaded gelatinase-stimuli nanoparticles in gelatinase-overexpressing BGC823 cells was significantly stronger than that in gelatinase-deficient GES-I cells. (B) After 24 hours of treatment with blank nanoparticles, free miR-200c, or miR-200c nanoparticles, we evaluated the expression of miR-200c in BGC823 and GES-I cells by real-time polymerase chain reaction assay. Free miR-200c had almost no effect on expression of intracellular miR-200c in BGC823 cells (I.3-fold over control) and GES-I cells (I.2-fold over control). In BGC823 cells, compared with the other groups, significant miR-200c overexpression (40.3-fold over control) was observed in the miR-200c nanoparticle groups. Meanwhile, in GES-I cells, only a 3.7-fold increase of miR-200c expression was observed in the miR-200c nanoparticle groups. Abbreviation: NPs, nanoparticles. 

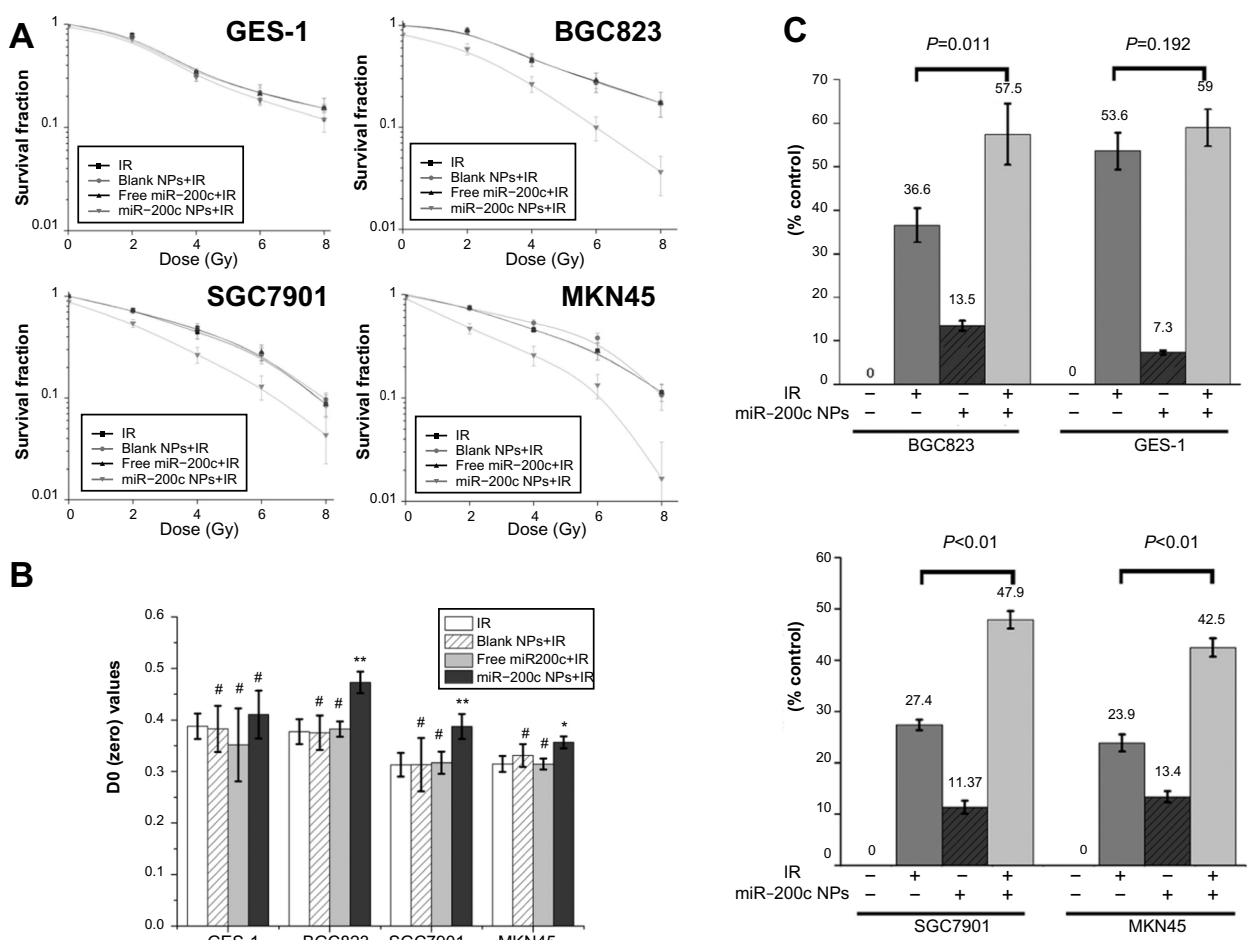

Figure 2 miR-200c nanoparticles achieved selective radiosensitization.

Notes: (A and B) The radiosensitization effects of blank nanoparticles, free miR-200c, and miR-200c nanoparticles were measured using the clonogenic survival assay. Cells were treated with subtoxic concentrations of miR-200c nanoparticles and equal concentrations of blank nanoparticles and free miR-200c for 24 hours and then exposed to increasing doses of ionizing radiation $(0,2,4,6$, and $8 \mathrm{~Gy})$. D0 values were calculated by a multitarget single-hit model $\left(S=I-\left(I-e^{-D / D 0}\right) N\right)$. The $S E R$ was calculated as the $D 0$ ratio between combination treatment and ionizing radiation alone. Cell survival curves obtained from the clonogenic survival assay are shown in (A). Cell survival curves for blank nanoparticles and free miR-200c in all the four cell lines almost overlapped with those for ionizing radiation alone. As shown in (B), blank nanoparticles and free miR-200c did not induce significant D0 increase ( $\left.{ }^{\# P}>0.05\right)$ over ionizing radiation alone groups in all the four cell lines. Compared with the ionizing radiation alone groups, D0 increased significantly in all three gastric cancer cell lines ( $* * P<0.01$ in BGC823 and SGC790I cells, *P< 0.05 in MKN45 cells). The SER value was I.25 for BGC823, I.24 for SGC790I, and I.I3 for MKN45, respectively. miR-200c nanoparticles did not cause a statistically significant D0 increase in GES-I (\#P>0.05), and the SER value was I.06. miR-200c nanoparticles significantly enhanced both ionizing radiation-induced cell clonogenic death and inhibition of cell proliferation $(P<0.05)$ in gastric cancer cells, but only slightly ( $\left.{ }^{*} P>0.05\right)$ in GES-I cells. (C) miR-200c nanoparticles sensitized BGC823 cells to 6 Gy ionizing radiation and an additional $20.9 \%$ ( $P=0.0 \mathrm{I}$ I) increased proliferation inhibition compared with cells exposed to ionizing radiation alone. Similar results were observed in SGC790I $(20.5 \%$, $* * P<0.0 \mathrm{I})$ and $\mathrm{MKN} 45$ cells $(\mathrm{I} 8.6 \%$, $* * P<0.0 \mathrm{I})$. However, the increase in GES-I cells was only $5.4 \%$ ( $P=0.192)$.

Abbreviations: NPs, nanoparticles; IR, ionizing radiation; SER, sensitizer enhancement ratio.

radiation + blank nanoparticle, and ionizing radiation + miR-200c nanoparticle groups, respectively. Compared with the control, the other groups showed significant differences $(P<0.01)$. Furthermore, compared with the other three groups, pretreatment with miR-200c nanoparticles significantly decreased the degree of cell invasiveness $(P<0.01)$.

We compared the expression of several critical apoptosisrelated proteins after treatment with miR-200c nanoparticles with that in the control group. We found that the expression of proapoptotic proteins, such as caspase-3, caspase-8, and caspase- 9 , was significantly higher in the group treated with miR-200c nanoparticles, whereas antiapoptotic protein Bcl-2 expression was significantly downregulated (Figure 4B). We then measured the percentage of apoptotic cells 72 hours after the treatments containing ionizing radiation. Compared with the control, the other groups showed significant differences $(P<0.01)$. Compared with the cell groups treated with ionizing radiation alone or a combination of blank nanopar- ticles and ionizing radiation, cells pretreated with miR-200c nanoparticles showed a significant increase in ionizing radiation-induced apoptosis (2.22-fold, $P<0.01$ for the ionizing radiation alone group; 2.07-fold, $P<0.01$ for the blank nanoparticles and ionizing radiation group). But compared with the ionizing radiation alone group, cells pretreated with blank nanoparticles did not significantly increase the ionizing radiation induced apoptosis ( $P>0.05$, Figure $4 \mathrm{C})$.

\section{miR-200c nanoparticles attenuated CSC- associated radioresistance mechanisms}

We detected basic and ionizing radiation-induced reactive oxygen species levels using $2^{\prime}, 7^{\prime}$-dichlorofluorescin diacetate. Treatment with miR-200c nanoparticles increased the prominent mean fluorescent intensity compared with controls (1.28-fold, $P<0.01$, Figure 5A). Compared with untreated controls, the groups treated with ionizing radiation showed a significant increase in mean fluorescent intensity $(P<0.01)$. 

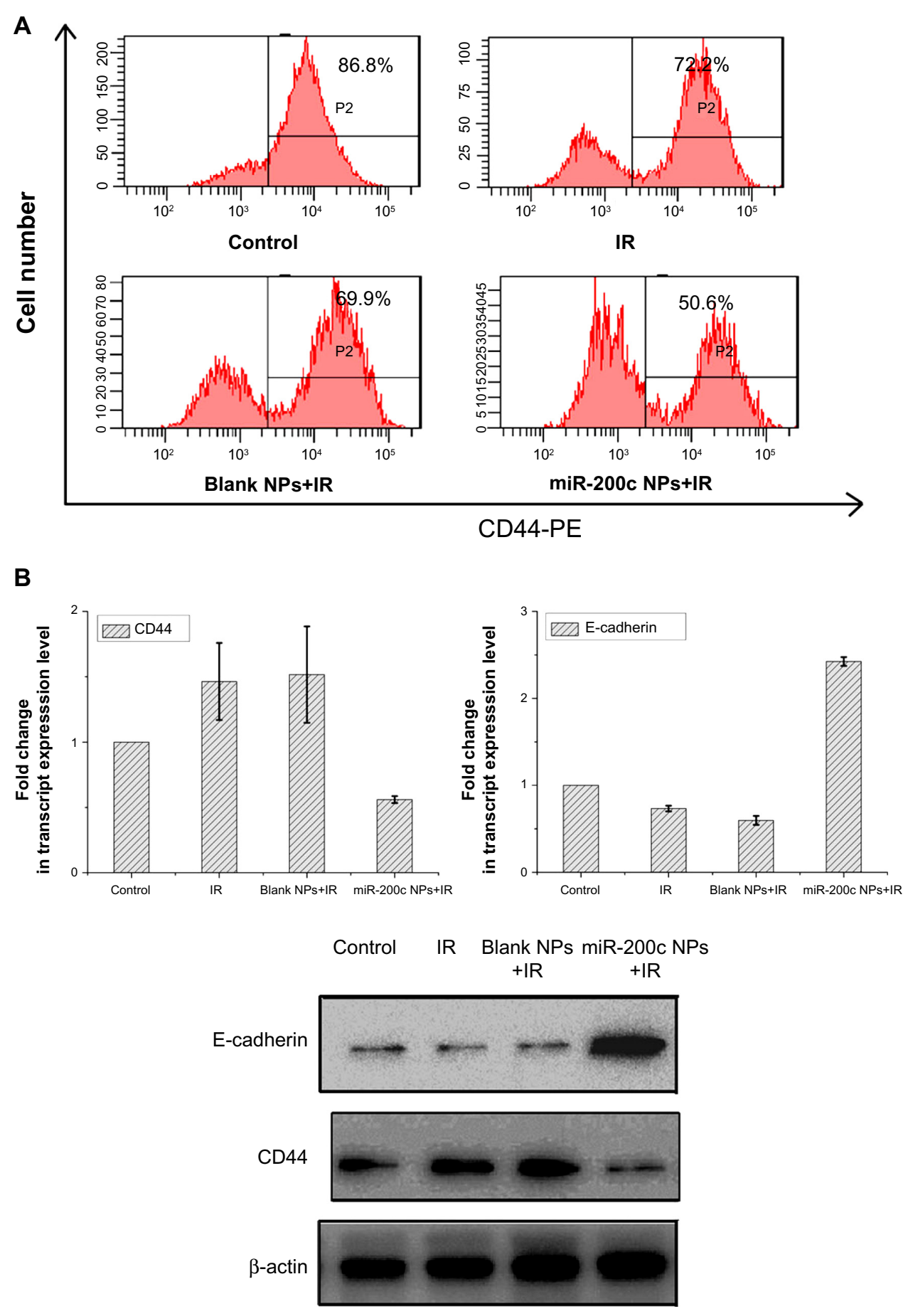

Figure 3 miR-200c nanoparticles affected cancer stem cell properties and the epithelial-mesenchymal transition process.

Notes: (A) Twenty-four hours after treatment, BGC823 cells were incubated with anti-CD44-PE and then analyzed by flow cytometry. The combination of miR-200c nanoparticles and ionizing radiation caused a reduction in the number of CD44+ cells. (B) mRNA and protein levels of CD44 and E-cadherin were also detected by real-time polymerase chain reaction and Western blot. The combination of miR-200c nanoparticles and ionizing radiation reduced CD44 expression and increased E-cadherin expression.

Abbreviations: NPs, nanoparticles; IR, ionizing radiation; PE, phycoerythrin.

More importantly, the mean fluorescent intensity of cells treated with a combination of miR-200c nanoparticles and ionizing radiation increased significantly compared with that in cells treated with ionizing radiation alone (1.15-fold, $P<0.01$, Figure 5B), confirming that miR-200c nanoparticles increased both basic and ionizing radiation-induced reactive oxygen species levels in BGC823 cells.

To investigate the induction and repair of ionizing radiationinduced DNA double strand breaks, $\gamma$-H2AX expression was measured at several time points post-ionizing radiation. For cells 
A

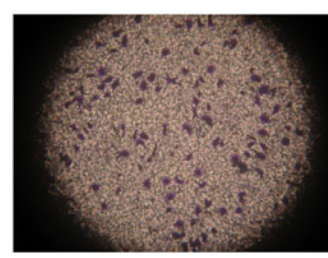

Control

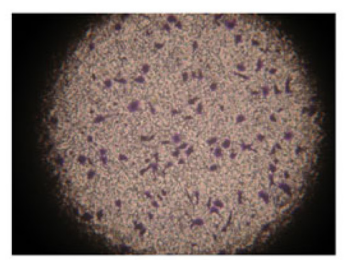

IR+blank NPs

B

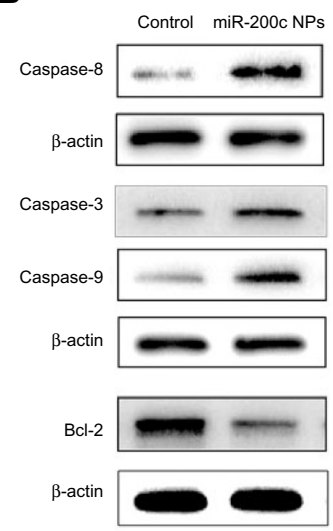

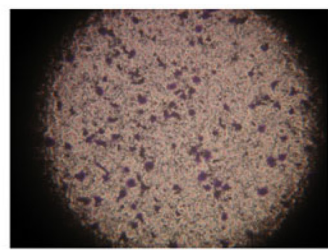

IR

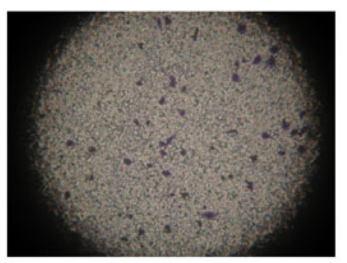

IR+miR-200c NPs

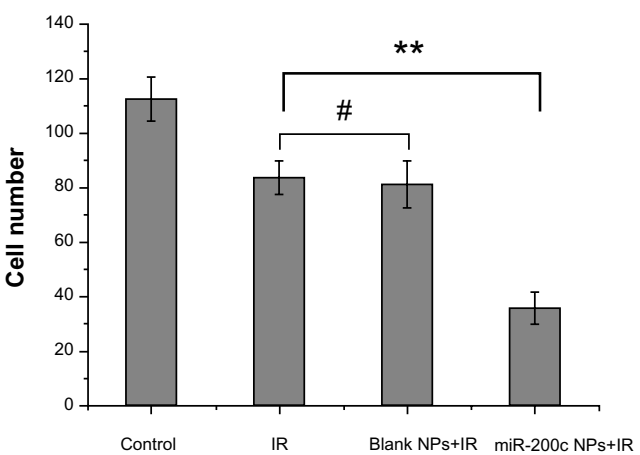

C

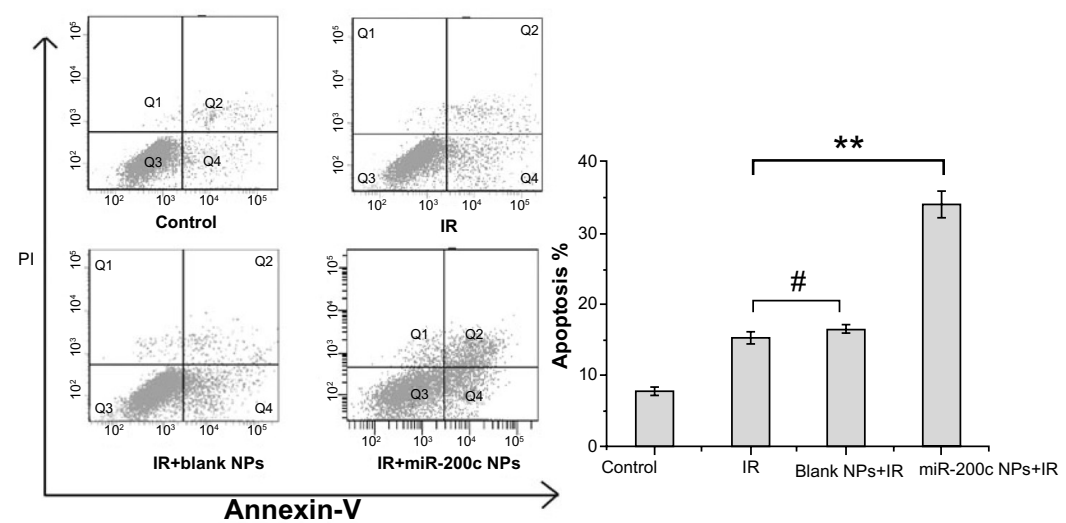

Figure 4 miR-200c nanoparticles suppressed cancer stem cell-like properties.

Notes: (A) The invasiveness of BGC823 cells was measured using the Transwell invasion assay. The cell numbers invading through the membrane into the lower compartment of the chamber were II $2.6 \pm 8.0,83.7 \pm 6.1,81.7 \pm 8.7$, and $35.7 \pm 6.0$ in the control, ionizing radiation, ionizing radiation + blank nanoparticle, and ionizing radiation + miR-200 $\mathrm{c}$ nanoparticle groups, respectively. Compared with the control group, the other groups showed significant differences $(P<0.0 \mathrm{I})$. Further, compared with the other three groups, the miR-200c nanoparticle + ionizing radiation combination group significantly decreased cell numbers invading through the membrane $(* * P<0.01)$. Compared with the ionizing radiation alone group, cells pretreated with blank nanoparticles did not significantly decrease the degree of cell invasiveness (\#P>0.05). (B) After 24 hours of treatment with miR-200c nanoparticles, expression of several apoptosis-related proteins in BGC823 cells was detected by Western blot. Compared with the untreated control, caspase-3, caspase-8, and caspase-9 levels were significantly higher in the miR-200c nanoparticle group, whereas the antiapoptotic Bcl-2 protein level was significantly downregulated. (C) Cell apoptosis was examined by costaining with annexin $V$ and propidium iodide. Compared with the control group, the other groups showed significant differences $(P<0.0 \mathrm{I})$. Compared with the group receiving ionizing radiation alone, BGC823 cells pretreated with miR-200c nanoparticles showed a significant increase in ionizing radiation induced-apoptosis $(2.22$-fold, ** $P<0.01)$. Compared with the ionizing radiation alone group, cells pretreated with blank nanoparticles did not significantly increase the ionizing radiation induced apoptosis ( ${ }^{*} P>0.05$ ).

Abbreviations: NPs, nanoparticles; IR, ionizing radiation.

treated with ionizing radiation alone, DNA double strand break repair was mostly complete by 16 hours after ionizing radiation. In contrast, when cells were treated with a combination of miR$200 \mathrm{c}$ nanoparticles and ionizing radiation, significantly higher expression of $\gamma$-H2AX was observed at 16 hours. Even at 24 hours, expression of $\gamma-\mathrm{H} 2 \mathrm{AX}$ could be detected (Figure 5C). Therefore, we concluded that miR-200c nanoparticles delay the repair of radiation-induced DNA double strand breaks.

\section{Discussion}

In previous work, we developed miR-200c-loaded nanoparticles based on a gelatinase-stimuli strategy, and evaluated their main characteristics. ${ }^{23}$ In this study, we investigated further whether miR-200c nanoparticles could act as radiosensitizers. Based on the abovementioned results, we demonstrated that the two main advantages of miR-200c nanoparticles are their selective therapeutic effect and significant radioenhancement via inhibition of CSC-like properties. The former benefited from the gelatinase-stimuli strategy and the latter could be attributed to the function of miR-200c.

Free miR-200c barely affected intracellular miR-200c expression and had no radiosensitization effect in BGC823 cells. In contrast, miR-200c was delivered into BGC 823 cells effectively by the nanoparticle formulation (with a 40.3 fold increase in miR-200c expression over control) and the miR-200c nanoparticles had a significant radioenhancement 
A
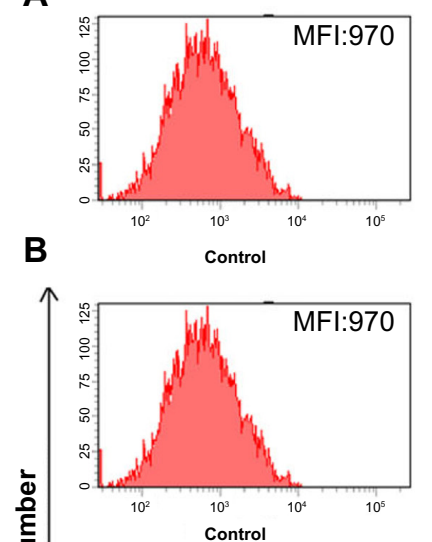

Control

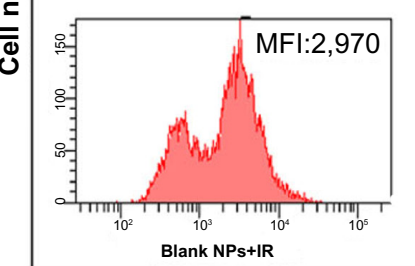

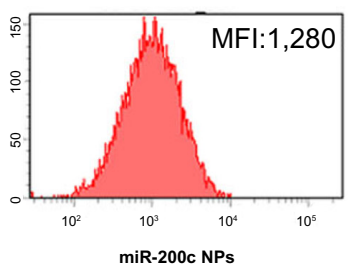
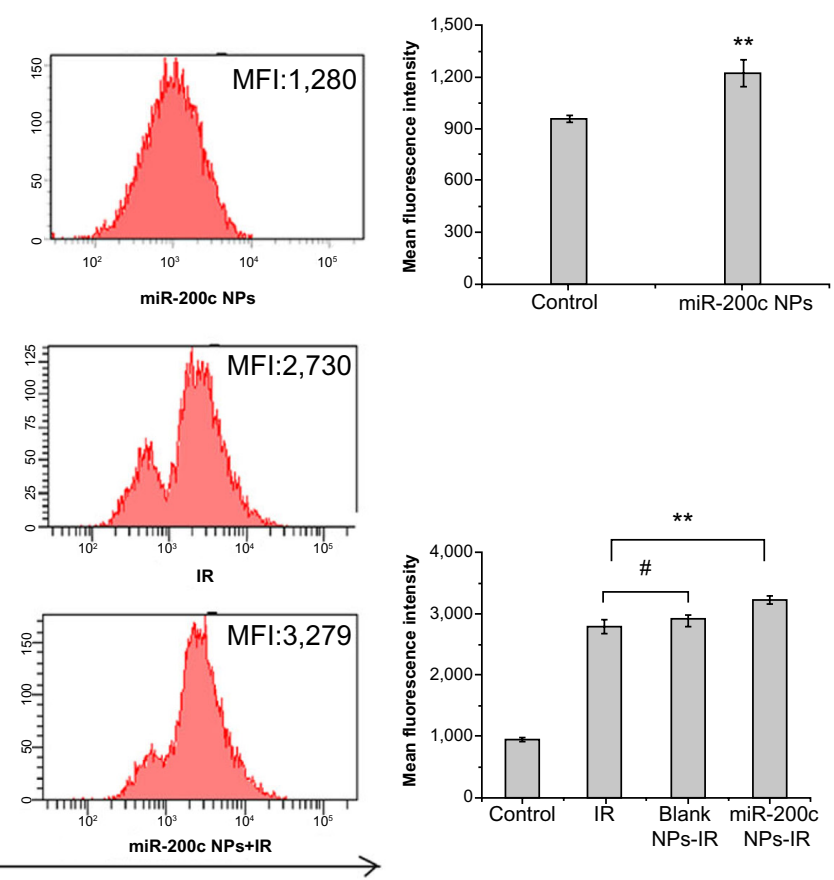

FITC

IR

C

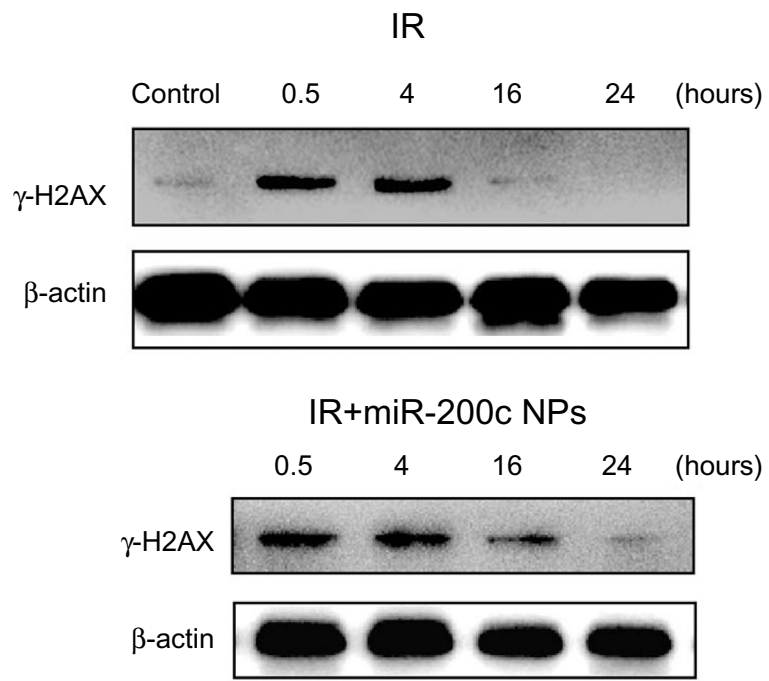

Figure 5 miR-200c nanoparticles impaired cancer stem cell-associated radioresistance mechanisms.

Notes: (A and B) Basic and ionizing radiation-induced reactive oxygen species levels were detected by flow cytometry using $2^{\prime}, 7^{\prime}$-dichlorofluorescin diacetate. Treatment with miR-200c nanoparticles resulted in an MFI increase over control (I.28-fold, $* * P<0.01)$. Compared with the untreated control, other ionizing radiation-containing groups showed a significant increase in MFI $(P<0.0 \mathrm{I})$. More importantly, compared with the ionizing radiation alone group, the MFI in the miR-200c nanoparticle + ionizing radiation group increased significantly $(1.15$-fold, $* * P<0.01)$. Compared with the ionizing radiation alone group, cells pretreated with blank nanoparticles did not significantly increase MFI ( $\left.{ }^{\#}>0.05\right)$. (C) $\gamma-\mathrm{H} 2 \mathrm{AX}$ expression was measured at several time points post-ionizing radiation to determine the induction and repair of ionizing radiation-induced DNA double strand breaks. DNA double strand break repair was almost complete 16 hours after treatment in cells receiving ionizing radiation alone. In contrast, significantly higher expression of $\gamma-\mathrm{H} 2 \mathrm{AX}$ was observed at 16 hours when treated with miR-200c nanoparticles + ionizing radiation; even at 24 hours, expression of $\gamma$ - $\mathrm{H} 2 \mathrm{AX}$ could be detected. Abbreviations: NPs, nanoparticles; IR, ionizing radiation; MFI, mean fluorescent intensity; FITC, fluorescein isothiocyanate.

effect. These data demonstrate that the gelatinase-stimuli nanoparticle formulation was an effective strategy for nucleic acid-based drug delivery.

Nonselective radiosensitization may increase ionizing radiation-induced injury to normal cells. The overlapping adverse reactions produced by ionizing radiation and a radiosensitizer will increase the risks associated with this therapeutic strategy and could even interrupt treatment. Selective radioenhancement effect was also achieved by the gelatinase-stimuli strategy. Gelatinases are generally overexpressed in a number of tumors but are almost undetectable in a nonpathological environment, ${ }^{27}$ and in previous studies we found that intracellular and excretory gelatinase levels were much higher in BGC823, SGC7901, and MKN45 
cells than in GES- 1 cells. ${ }^{26}$ We have already confirmed that PEG-Pep-PCL conjugates can be cleaved by gelatinases. ${ }^{21}$ The resulting non-PEGylated nanoparticles have a higher affinity for cells than PEGylated nanoparticles, causing greater intracellular drug accumulation. This improved cellular uptake of nanoparticles depends on the cellular gelatinase expression level. In the present cellular uptake and PCR assays, selective miR-200c nanoparticle uptake efficiency was seen between cancer cells and normal cells. The final selective radioenhancement effect is probably attributable to this selective cellular uptake.

miR-200c was effectively and selectively delivered by gelatinase-stimuli nanoparticles, and our results show that the significant radioenhancement achieved was closely associated with inhibition of CSC-like properties. Firstly, we detected the impact of miR-200c nanoparticles on gastric cancer stem cell markers, which are presently thought to include CD44. Takaishi et $\mathrm{al}^{6}$ found that CD44 was a potential marker for gastric CSCs by screening a series of potential CSC markers in various human gastric cancer cell lines. $\mathrm{CD}_{4} 4^{+}$gastric cancer cells showed spheroid colony formation properties in vitro and tumorigenicity in vivo, but CD44- gastric cancer cells did not. Further, $\mathrm{CD} 44^{+}$gastric cancer cells showed much greater resistance to chemotherapy and radiotherapy than $\mathrm{CD}^{-} 4^{-}$gastric cancer cells, Also, in $\mathrm{CD}_{4}{ }^{+}$gastric cancer cells, high CD44-expressing cells showed significant superiority in both spheroid colony formation properties and tumorigenicity than low CD44-expressing cells. These authors also found some gastric cancer cell lines (MKN-45 and MKN-74) had a sizeable subpopulation of CD44+ cells (up to 94\%). We found that BGC823 cells also had high CD44 expression (up to $86.8 \%$ ) and that treatment with a combination of miR-200c nanoparticles and ionizing radiation reduced the percentage of $\mathrm{CD}_{4} 4^{+}$cells and $\mathrm{CD} 44$ mRNA and protein expression in BGC823 cells. Therefore, the radiosensitization effect of miR-200c nanoparticles in gastric cancer cells was associated with the reduction of the percentage expressing CD44 and the total expression level. It has been reported that emergence of CSCs is partly due to EMT. ${ }^{17}$ Several studies have also reported a relationship between EMT and radioresistance. ${ }^{18,28}$ Our results indicate that the combination of miR-200c nanoparticles and ionizing radiation increases expression of E-cadherin, an important epithelial marker, and could reverse the EMT process. Our experiments further confirmed that two main properties of CSCs, ie, invasiveness and resistance to apoptosis, and two main mechanisms involved in CSC radioresistance, ie, reactive oxygen species generation and DNA double strand break repair, were impaired by the miR-200c nanoparticles, suggesting that radiosensitization of miR-200c nanoparticles is dependent on its ability to inhibit CSCs.

Our present results suggest that miR-200c nanoparticles have some potential advantages that warrant further investigation. Firstly, CSCs and normal stem cells share many common characteristics, and CSC-targeted therapies tend to simultaneously injure normal stem cells. It has been reported that members of the miR-200 family can also influence generation of normal stem cells. ${ }^{29}$ Our miR-200c nanoparticles could overcome this problem to some extent by the gelatinase-dependent difference in uptake between cancer cells and normal cells. Secondly, overexpression of gelatinase was associated with radioresistance ${ }^{30}$ and CSCs. ${ }^{31}$ Cellular uptake of gelatinase-stimuli nanoparticles depends on cellular expression of gelatinase. Therefore, the gelatinasestimuli strategy had the potential to target nanoparticles more effectively to these radioresistant cell populations. Thirdly, the adverse effects of radiotherapy, especially ionizing radiation-induced invasion, are of increasing concern..$^{32-34}$ Bao et $\mathrm{al}^{4}$ and Phillips et $\mathrm{al}^{7}$ have reported that ionizing radiation enriches the CSC-like subpopulation. It was also reported that ionizing radiation could prompt cancer cells to undergo EMT. ${ }^{35}$ These reports suggest that, CSCs and EMT were associated with ionizing radiation induced invasion. Tumor metastasis in spite of successful local control by ionizing radiation is encountered in many patients. The combination of anti-invasion drugs and ionizing radiation may improve the clinical outcomes in such cases. miR-200cloaded nanoparticles are agents that can potentially decrease ionizing radiation-induced invasion by simultaneously suppressing CSCs and EMT. Moreover, gelatinases that degrade the extracellular matrix are closely related to the invasiveness of cancer cells. ${ }^{27}$ The gelatinase-stimuli strategy enables nanoparticles to be better targeted to cancer cells with high metastatic potential.

Certain miRNAs have been confirmed to have important roles in the response to ionizing radiation..$^{36}$ However, the effect of therapeutic miRNAs and ionizing radiation used in combination has not been well studied. Ionizing radiation may further influence the unstable structure and function of miRNAs. Due to their unique properties, miRNA-loaded nanoparticles have the potential to overcome this problem. Meanwhile, by using a targeting strategy, nanoparticles can further improve the efficacy of tumor-specific drug delivery beyond enhanced permeability and retention-mediated tumor homing. Nanomedicine with a targeting strategy will have the greatest impact when used as a radiosensitizer. ${ }^{37}$ In this 
study, we detected the radiosensitization effect of miR-200c in gastric cancer cells. Our results demonstrate that miR-200c-loaded nanoparticles enhance the effects of radiotherapy effect for the following reasons:

- efficient delivery of miRNA, with enhanced miRNA stability and cellular uptake

- tumor-targeted delivery of miRNAs as a radiosensitizer, exploiting the difference between the microenvironments of tumor cells and normal cells, achieving the maximal benefits of radiotherapy with regard to injuring cancer cells while sparing normal cells

- CSC-related radiosensitization, increasing the radiosensitivity of cancer cells by impairing the two main mechanisms involving in radioresistance of CSCs

- reducing invasiveness, with the potential to decrease ionizing radiation-induced invasion

- better targeting of nanoparticles to cell subpopulations with radioresistance and high metastatic potential via the gelatinase-stimuli strategy.

To our knowledge, this is the first study demonstrating the radioenhancement potential of miR-200c in gastric cancer and the radiosensitization was associated with its CSCs inhibitory function. In vivo research based on subcutaneous transplanted tumor models and even models of human gastric carcinoma involving orthotopic transplantation is now needed. More studies also need to be done in other tumor types to confirm further the superiority of miR-200c-loaded nanoparticles with regard to radioenhancement.

\section{Conclusion}

Our study demonstrates that miR-200c nanoparticles were effective radiosensitizers in gelatinase-overexpressing gastric cancer cells but had little radiosensitization in gelatinasedeficient normal gastric mucosa cells. The radioenhancement in gastric cancer cells was associated with suppressing CSClike properties and impairing CSCs associated radioresistance mechanisms. In conclusion, miR-200c nanoparticles were highly selective and effective radiosensitizers in gastric cancer by inhibiting CSC-like properties and the EMT process, making them a potentially superior platform for further preclinical and clinical evaluation.

\section{Acknowledgments}

This work was supported by the National Natural Science Foundation of China $(81071815,81172281,81220108023$, $81101751,81101816,81302053)$, the Jiangsu Provincial Special Program of Medical Science (BL2012001), the Nanjing Medical Science and Technique Development
Foundation (Youth Foundation Level 3), and the Nanjing Medical Science and Technique Development Foundation (Outstanding Youth Foundation, JQX12003). We thank Mr Qiu for his scientific contribution to this work.

\section{Disclosure}

The authors report no competing interests exist in this work.

\section{References}

1. Yeh JM, Kuntz KM, Ezzati M, Hur C, Kong CY, Goldie SJ. Development of an empirically calibrated model of gastric cancer in two high-risk countries. Cancer Epidemiol Biomarkers Prev. 2008;17(5):1179-1187.

2. Hartgrink HH, Jansen EP, van Grieken NC, van de Velde CJ. Gastric cancer. Lancet. 2009;374(9688):477-490.

3. Diehn M, Cho RW, Lobo NA, et al. Association of reactive oxygen species levels and radioresistance in cancer stem cells. Nature. 2009;458(7239):780-783.

4. Bao S, Wu Q, McLendon RE, et al. Glioma stem cells promote radioresistance by preferential activation of the DNA damage response. Nature. 2006;444(7120):756-760.

5. Baumann M, Krause M, Thames H, Trott K, Zips D. Cancer stem cells and radiotherapy. Int $J$ Radiat Biol. 2009;85(5):391-402.

6. Takaishi S, Okumura T, Tu S, et al. Identification of gastric cancer stem cells using the cell surface marker CD44. Stem Cells. 2009;27(5):1006-1020.

7. Phillips TM, McBride WH, Pajonk F. The response of CD24(-/low)/ CD44+ breast cancer-initiating cells to radiation. J Natl Cancer Inst. 2006;98(24):1777-1785.

8. Chiou SH, Kao CL, Chen YW, et al. Identification of CD133-positive radioresistant cells in atypical teratoid/rhabdoid tumor. PLoS One. 2008;3(5):e2090.

9. de Jong MC, Pramana J, van der Wal JE, et al. CD44 expression predicts local recurrence after radiotherapy in larynx cancer. Clin Cancer Res. 2010;16(21):5329-5338.

10. Yaromina A, Krause M, Thames H, et al. Pre-treatment number of clonogenic cells and their radiosensitivity are major determinants of local tumour control after fractionated irradiation. Radiother Oncol. 2007;83(3):304-310.

11. Ponnurangam S, Mammen JM, Ramalingam S, et al. Honokiol in combination with radiation targets notch signaling to inhibit colon cancer stem cells. Mol Cancer Ther. 2012;11(4):963-972.

12. Kim WK, Kim JH, Yoon K, et al. Salinomycin, a p-glycoprotein inhibitor, sensitizes radiation-treated cancer cells by increasing DNA damage and inducing G2 arrest. Invest New Drugs. 2012;30(4): 1311-1318.

13. Shimono Y, Zabala M, Cho RW, et al. Downregulation of miRNA200c links breast cancer stem cells with normal stem cells. Cell. 2009;138(3):592-603.

14. Lim YY, Wright JA, Attema JL, et al. Epigenetic modulation of the miR-200 family is associated with transition to a breast cancer stem-cell-like state. J Cell Sci. 2013;126(Pt 10):2256-2266.

15. Lo WL, Yu CC, Chiou GY, et al. MicroRNA-200c attenuates tumour growth and metastasis of presumptive head and neck squamous cell carcinoma stem cells. J Pathol. 2011;223(4):482-495.

16. Park SM, Gaur AB, Lengyel E, Peter ME. The miR-200 family determines the epithelial phenotype of cancer cells by targeting the E-cadherin repressors ZEB1 and ZEB2. Genes Dev. 2008;22(7): 894-907.

17. Polyak K, Weinberg RA. Transitions between epithelial and mesenchymal states: acquisition of malignant and stem cell traits. Nat Rev Cancer. 2009;9(4):265-273. 
18. Theys J, Jutten B, Habets R, et al. E-cadherin loss associated with EMT promotes radioresistance in human tumor cells. Radiother Oncol. 2011;99(3):392-397.

19. Floor S, van Staveren WC, Larsimont D, Dumont JE, Maenhaut C. Cancer cells in epithelial-to-mesenchymal transition and tumorpropagating-cancer stem cells: distinct, overlapping or same populations. Oncogene. 2011;30(46):4609-4621.

20. Muthiah M, Park IK, Cho CS. Nanoparticle-mediated delivery of therapeutic genes: focus on miRNA therapeutics. Expert Opin Drug Deliv. 2013;10(9):1259-1273.

21. Liu Q, Li RT, Qian HQ, et al. Gelatinase-stimuli strategy enhances the tumor delivery and therapeutic efficacy of docetaxel-loaded poly(ethylene glycol)-poly(varepsilon-caprolactone) nanoparticles. Int J Nanomedicine. 2012;7:281-295.

22. Li R, Wu W, Liu Q, et al. Intelligently targeted drug delivery and enhanced antitumor effect by gelatinase-responsive nanoparticles. PLoS One. 2013;8(7):e69643.

23. Liu Q, Li RT, Qian HQ, et al. Targeted delivery of miR-200c/DOC to inhibit cancer stem cells and cancer cells by the gelatinases-stimuli nanoparticles. Biomaterials. 2013;34(29):7191-7203.

24. Werner ME, Copp JA, Karve S, et al. Folate-targeted polymeric nanoparticle formulation of docetaxel is an effective molecularly targeted radiosensitizer with efficacy dependent on the timing of radiotherapy. ACS Nano. 2011;5(11):8990-8998.

25. Liu N, Tang LL, Sun Y, et al. MiR-29c suppresses invasion and metastasis by targeting TIAM1 in nasopharyngeal carcinoma. Cancer Lett. 2013;329(2):181-188.

26. Cui FB, Li RT, Liu Q, et al. Enhancement of radiotherapy efficacy by docetaxel-loaded gelatinase-stimuli PEG-Pep-PCL nanoparticles in gastric cancer. Cancer Lett. 2014;346(1):53-62.

27. Gupta GP, Massague J. Cancer metastasis: building a framework. Cell. 2006;127(4):679-695.

28. Kurrey NK, Jalgaonkar SP, Joglekar AV, et al. Snail and slug mediate radioresistance and chemoresistance by antagonizing p53-mediated apoptosis and acquiring a stem-like phenotype in ovarian cancer cells. Stem Cells. 2009;27(9):2059-2068.
29. Wang G, Guo X, Hong W, et al. Critical regulation of miR-200/ZEB2 pathway in Oct4/Sox2-induced mesenchymal-to-epithelial transition and induced pluripotent stem cell generation. Proc Natl Acad Sci USA. 2013;110(8):2858-2863.

30. Cordes N, van Beuningen D. Cell adhesion to the extracellular matrix protein fibronectin modulates radiation-dependent G2 phase arrest involving integrin-linked kinase (ILK) and glycogen synthase kinase3 beta (GSK-3beta) in vitro. Br J Cancer. 2003;88(9):1470-1479.

31. Takebe N, Harris PJ, Warren RQ, Ivy SP. Targeting cancer stem cells by inhibiting Wnt, Notch, and Hedgehog pathways. Nat Rev Clin Oncol. 2011;8(2):97-106.

32. Wild-Bode C, Weller M, Rimner A, Dichgans J, Wick W. Sublethal irradiation promotes migration and invasiveness of glioma cells: implications for radiotherapy of human glioblastoma. Cancer Res. 2001;61(6):2744-2750.

33. Kaliski A, Maggiorella L, Cengel KA, et al. Angiogenesis and tumor growth inhibition by a matrix metalloproteinase inhibitor targeting radiation-induced invasion. Mol Cancer Ther. 2005;4(11):1717-1728.

34. Qian LW, Mizumoto K, Urashima T, et al. Radiation-induced increase in invasive potential of human pancreatic cancer cells and its blockade by a matrix metalloproteinase inhibitor, CGS27023. Clin Cancer Res. 2002;8(4):1223-1227.

35. Zhou YC, Liu JY, Li J, et al. Ionizing radiation promotes migration and invasion of cancer cells through transforming growth factor-betamediated epithelial-mesenchymal transition. Int J Radiat Oncol Biol Phys. 2011;81(5):1530-1537.

36. Czochor JR, Glazer PM. microRNAs in cancer cell response to ionizing radiation. Antioxid Redox Signal. February 4, 2014. [Epub ahead of print.]

37. Chow EK, Ho D. Cancer nanomedicine: from drug delivery to imaging. Sci Transl Med. 2013;5(216):216rv4. 


\section{Supplementary material \\ The cytotoxicity of miR-200c nanoparticles}

Four kinds of cells were treated with blank nanoparticles (NPs), free miR-200c or miR-200c NPs for 24 hours, and then cell viability was measured by the MTT assay to determine subtoxic concentrations of miR-200c NPs (Figure S1).

Firstly, we found that blank NPs almost did not show cytotoxicity to all four kinds of cells (cell proliferation inhibition $<5 \%$ ) in the tested concentration range, indicating the copolymers were almost nontoxic to tested cells.
miR-200c NPs caused cytotoxicity in all kinds of cells in a dose-dependent manner. Free miR-200c induced much lower cytotoxicity than miR-200c NPs at each concentration. Obtained lower concentrations of miR-200c NPs that induced slight cytotoxicity (cell proliferation inhibition $<15 \%$ ) were used as subtoxic concentrations for subsequent planned experiments. This was a prerequisite for studying radiosensitization and to ensure that the subsequent findings did not attribute to a direct cytotoxic effect. The used concentrations were $128 \mu \mathrm{mol} / \mathrm{L}$ for both BGC823 and GES-1, $512 \mu \mathrm{mol} / \mathrm{L}$ for SGC7901, and $256 \mu \mathrm{mol} / \mathrm{L}$ for MKN45, respectively.
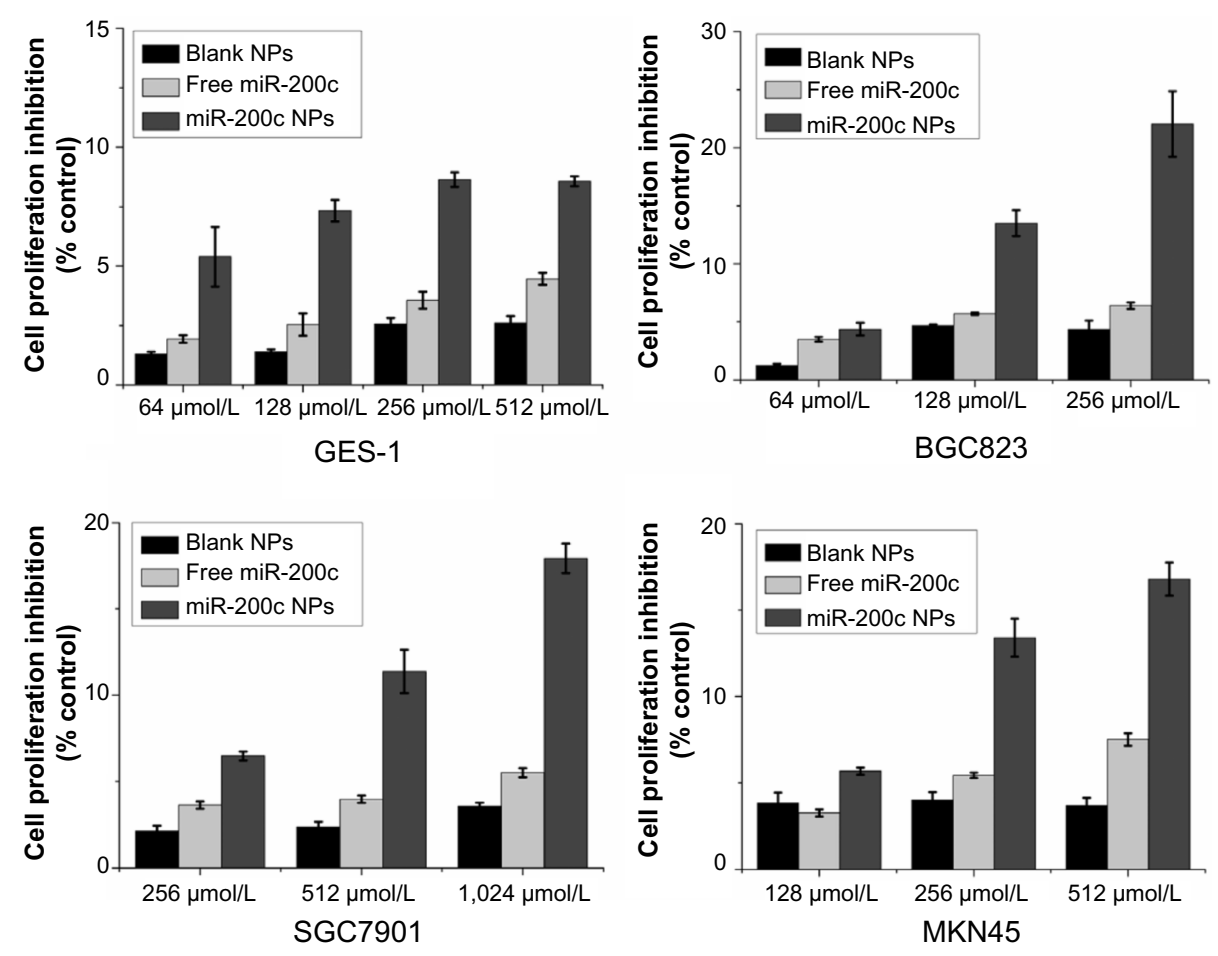

Figure SI Inhibition of cell proliferation by miR-200c nanoparticles on four types of cells. Abbreviation: NPs, nanoparticles.

$$
\begin{aligned}
& \text { Plating effciency }(\%)=\frac{\text { Number of colonies formed }}{\text { Number of cells seeded }} \times 100 \\
& \text { Surviving fraction }(\%)=\frac{\text { Number of colonies formed after treatment }}{\text { Number of cells seeded } \times P E} \times 100 \\
& \text { Sensitizer enhancement ratio }(\%)=\frac{D 0 \text { value of drug }+I R \text { group }}{D 0 \text { value of IR alone group }}
\end{aligned}
$$

Figure S2 Calculations of plating efficiency, surviving fraction and the sensitizer enhancement ratio.

Notes: D0 values were calculated by a multitarget single-hit model $\left(S=I-\left(I-e^{D / D 0}\right) N\right)$. Sensitizer enhancement ratio was calculated as a $D 0$ ratio between combination treatment and IR alone.

Abbreviations: IR, ionizing radiation; PE, plating efficiency. 


\section{E-cadherin (forward 5'-GCTCTTGGCTGTGCAGATTA-3', reverse 5'-ACGGTTATACCCAGCAGTCC-3') \\ CD44 (forward 5'-CTGGGTCCCTCTTCAACACT-3', reverse 5'-TGTTCTAGGGACCACGTTGA-3') \\ $\beta$-actin (forward 5'-TTCCTGGGCATGGAGTC-3', reverse 5'-CAGGTCTTTGCGGATGTC-3')}

Figure S3 Primer specific sequences for E-cadherin, CD44 and $\beta$-actin control in the SYBR ${ }^{\circledR}$ Green real time PCR.

Abbreviation: PCR, polymerase chain reaction.

\section{Publish your work in this journal}

The International Journal of Nanomedicine is an international, peerreviewed journal focusing on the application of nanotechnology in diagnostics, therapeutics, and drug delivery systems throughout the biomedical field. This journal is indexed on PubMed Central, MedLine, CAS, SciSearch $\AA$, Current Contents ${ }^{\circledR} /$ Clinical Medicine,
Journal Citation Reports/Science Edition, EMBase, Scopus and the Elsevier Bibliographic databases. The manuscript management system is completely online and includes a very quick and fair peer-review system, which is all easy to use. Visit http://www.dovepress.com/ testimonials.php to read real quotes from published authors. 